\title{
Thermal-Initiating Potentialities of Poly(methyl methacrylate) Peroxide: Metamorphosis of Block-into-Block Copolymer and Comparative Studies on Surface Texture and Morphology
}

\author{
AJAYA KUMAR NANDA, ${ }^{1}$ KISHORE $^{2}$ \\ ${ }^{1}$ Department of Inorganic and Physical Chemistry, Indian Institute of Science, Centre of Advanced Study, \\ Bangalore-560012, India \\ 2 Polymer Composites Laboratory, Department of Metallurgy, Indian Institute of Science, Bangalore-560012, India
}

\begin{abstract}
This is the first report concerning the use of vinyl polyperoxide, namely, poly(methyl methacrylate) peroxide (PMMAP), as a thermal initiator for the synthesis of active polymer PMMAP-PS-PMMAP by free-radical polymerization with styrene. The polymerizations have been carried out at different concentrations of macroinitiator PMMAP. The active polymers have been characterized by ${ }^{1} \mathrm{H}$ NMR, DSC, thermogravimetric analysis, and gel permeation chromatography. PMMAP-PS-PMMAP is further used as the thermal macroinitiator for the preparation of another block copolymer, PMMA-b-PS-bPMMA, by reacting the active polymers with methyl methacrylate. The block copolymers have been synthesized by varying the concentrations of the active polymers. The mechanism of block copolymers has been discussed, which is also supported by thermochemical calculations. Studies on the surface texture and morphology of the block copolymer of polystyrene (PS) and PMMA material have been carried out using scanning electron microscopy. Furthermore, in this article, a blend of the same constituent materials (PS and PMMA) in proportions (v/v) similar to that contained in block copolymers has been formulated, and the morphology and surface textures of these materials were also investigated. A comparative microscopical evaluation between two processing methods was done for a better understanding of the processing route dependence of the microstructures. () 2001 John Wiley \& Sons, Inc. J Polym Sci A: Polym Chem 39: 546-554, 2001
\end{abstract}

Keywords: macroinitiator; active polymer; block copolymer; morphological characterization

\section{INTRODUCTION}

Polymer alloys and blends are gaining the increasing attention of research workers as they can be

Correspondence to: A. K. Nanda (E-mail: ajaya@ipc. suitably processed to yield tailormade properties to meet the stringent demands in technologically advanced applications. ${ }^{1}$ Recently, there is a growing interest in the usage of block copolymers in varied fields like polymer alloys (blends), modifications, compatibility improvements, and surface modifications, to name a few. ${ }^{2-5}$ Furthermore, the surface texture and morphological features of these block copolymers and blends have attracted the attention 
of microscopists because this approach clearly provides an idea about the physical arrangement of different constituents present in the materials. ${ }^{6-13}$

Unlike simple low molecular weight peroxides, their polymeric counterparts, although long known, have not been studied extensively with respect to their initiating characteristics. ${ }^{14,15}$ Among polymeric peroxides, vinyl polyperoxides $^{15}$ play a primary role because they are obtained via radical, alternating copolymerization reactions of vinyl monomers and oxygen. Vinyl polyperoxides are attractive candidates owing to their divergent characteristics of exothermic degradation, ${ }^{16-18}$ autopyrolysis, ${ }^{19}$ special fuels, ${ }^{19,20}$ autoacceleration, ${ }^{21}$ curatives in coating and molding applications, ${ }^{22}$ and finally their use as baseassisted initiators. ${ }^{23}$ Vinyl polyperoxides are superior to acid polyperoxides ${ }^{24-26}$ on the basis of their high solubility in vinyl monomers, safety in handling, economy, and simplicity of synthesis.

This is the first report on the use of poly(methyl methacrylate) peroxide (PMMAP) as a thermal initiator for the polymerization of styrene resulting in the synthesis of active polymers. The metamorphosis of a block-into-block copolymer has been carried out since this aspect appears to be quite interesting and less explored. ${ }^{27,28}$ To widen the scope of the present investigation, an attempt has been made to study and then characterize the morphological features of the constituent materials first and thereafter examine the surface texture of the block copolymer using scanning electron microscopy (SEM). To make the microscopy part of the work more comprehensive, blends of the same constituent phases in proportions $(\mathrm{v} / \mathrm{v})$ similar to those seen in block copolymers were made, and the texture and morphological features of these blends were also studied. Based on these efforts, a comparative evaluation has been attempted in this article. A preliminary investigation to correlate processing-route-dependence of the microstructure in these polymeric systems has also been initiated.

\section{EXPERIMENTAL SECTION}

\section{Materials}

Methyl methacrylate (MMA) and styrene (STY) were purified from the inhibitor by washing with $10 \% \mathrm{NaOH}$ solution and then with water several times. After drying over anhydrous $\mathrm{Na}_{2} \mathrm{SO}_{4}$, MMA and STY were distilled under reduced pres- sure. The Cobalt(II) tetraphenylporphyrin [Co(II)TPP] (Aldrich) was used as received. Methanol, petroleum ether, chloroform, cyclohexane, acetonitrile, ethyl acetate, benzene, chlorobenzene, and pyridine (Py) were purified by adopting established procedures. ${ }^{29}$

\section{Preparation of Co(II)TPP(Py) Complex}

$\mathrm{Co}(\mathrm{II}) \mathrm{TPP}(\mathrm{Py})$ complex was prepared by mixing solution of equivalent concentrations $\left(1.0 \times 10^{-4}\right.$ molś $\mathrm{L}^{-1}$ ) of Co(II)TPP and Py, respectively, in MMA. The formation of Co(II)TPP(Py) has been confirmed by the bathochromic shift of 413 (soret band) and $528 \mathrm{~nm}$ (Q band) of Co(II)TPP complex to 440 and $553 \mathrm{~nm}$, respectively, in the UV-vis absorption spectra.

\section{Synthesis of PMMAP}

$\mathrm{Co}$ (II)TPP(Py) $\left(1.0 \times 10^{-4} \mathrm{~mol} \cdot \mathrm{L}^{-1}\right)$ in MMA (8.4 $\mathrm{mol} \cdot \mathrm{L}^{-1}$ ) solution was placed in a $300-\mathrm{mL}$ Parr reactor (Parr Instrument Co.) and pressurized at 200 psi of oxygen pressure. The polymerization was carried out at $50{ }^{\circ} \mathrm{C}$ with constant stirring for $8 \mathrm{~h}$, and the polyperoxide was precipitated from the reaction mixture using petroleum ether as a nonsolvent. Thus, the PMMAP obtained was purified by repeated precipitation from chloroform solution and dried in vacuum to a constant weight.

\section{Synthesis of PMMAP-PS-PMMAP "Active" Polymer}

The preparation of polystyrene (PS) involved the use of macroinitiators PMMAP and STY at $70{ }^{\circ} \mathrm{C}$ for $3 \mathrm{~h}$ in Pyrex ampoules, which were previously sealed after repeated freeze-thaw cycles in liquid nitrogen. The polymerizations were carried out at various concentrations of PMMAP. Following this, the active polymers PMMAP-PS-PMMAP were precipitated using methanol as a nonsolvent, purified by repeated precipitation from chloroform solution, and dried in vacuum to a constant weight. The PMMAP that remained was removed by precipitating the polymers twice in petroleum ether/ethyl acetate $(1: 1)$ followed by methanol. The active polymer PMMAP-PS-PMMAP containing peroxide units in the main chain was collected.

Synthesis of Poly(methyl methacrylate)-BlockPolystyrene-Block-Poly(methyl methacrylate)

The PMMAP-PS-PMMAP active polymer as well as MMA were taken in a Pyrex ampoule, which 
was previously sealed after repeated freeze-thaw cycles in liquid nitrogen, and thermostated ${ }^{30}$ at $75{ }^{\circ} \mathrm{C}$ for $5 \mathrm{~h}$. The polymerizations were carried out at various concentrations of active polymers. The polymer, poly(methyl methacrylate)-block-polystyrene-block-poly(methyl methacrylate) (PMMA-bPS-b-PMMA), obtained was precipitated over methanol and purified by repeated precipitation from chloroform solution and dried at $50{ }^{\circ} \mathrm{C}$ in vacuum to a constant weight.

\section{Purification of PMMA-b-PS-b-PMMA}

The STY and MMA homopolymers, made as byproducts in the preparations of PMMA-b-PS-bPMMA, were purified by solvent extractions. About $2 \mathrm{~g}$ of polymer product were extracted with cyclohexane for $50 \mathrm{~h}$ by a Soxhlet extractor to extract PS using cylindrical filter paper. The dried residue was treated with acetonitrile for $50 \mathrm{~h}$ to extract PMMA. The remaining polymer was extracted with benzene for $50 \mathrm{~h}$ to extract PMMA-b-PS-b-PMMA. Each extracted solution was concentrated by evaporating the excess solvent using a rotary evaporator (Yamato Hi Tec, Model RE-51), and they were added to $300 \mathrm{~mL}$ of methanol to precipitate out each polymer.

\section{Synthesis of PS and PMMA Blend}

The blend was prepared by dissolving $75: 25(\mathrm{v} / \mathrm{v})$ of PMMA and PS constituents, respectively, in $\mathrm{CHCl}_{3}$ followed by precipitation with methanol $^{31,32}$ and dried under vacuum to a constant weight.

\section{Analytical Techniques}

A Bruker ACF 200-MHz model spectrometer was used to record the ${ }^{1} \mathrm{H}$ NMR spectra using $\mathrm{CDCl}_{3}$ as a solvent. The chemical shifts were assigned with reference to tetramethylsilane. Thermal analysis was done on a Perkin-Elmer DSC-2C at a heating rate of $10{ }^{\circ} \mathrm{C} / \mathrm{min}$ under nitrogen atmosphere. Thermogravimetric analysis (TGA) was carried out on a Perkin-Elmer TGS-2 instrument under nitrogen atmosphere at a heating rate of 10 ${ }^{\circ} \mathrm{C} / \mathrm{min}$. The number average molecular weights $\left(\bar{M}_{n}\right)$ and weight average molecular weights $\left(\bar{M}_{w}\right)$ and polydispersity index $\left(\bar{M}_{w} / \bar{M}_{n}\right)$ were estimated using gel permeation chromatography (GPC). GPC was performed on a modular system comprising of a Waters 590 high-performance liquid chromatography pump, a Waters 717 autosam- pler (Waters Corp., Milford, MA), and an ERMA ERC-7515A refractive index detector (ERMA CR, Inc., Tokyo). The column used was a $60-\mathrm{cm}$ PLGel mixed-B $10-\mu \mathrm{m}$ column (Polymer Laboratories Ltd., Shropshire, UK). The mobile phase used was unstabilized tetrahydrofuran (EM Science, Gibbstown, NJ) at a flow rate of $0.95 \mathrm{~mL} / \mathrm{min}$. The molecular weights were calculated using 13 narrow PS standards from 6,300,000 to 580 (Pressure Chemical Co., Pittsburgh, PA). The software used for the calculations was Polymer Laboratories Ltd. caliber version 7.04 (Polymer Laboratories Ltd.).

\section{SEM Microscopy}

The samples processed, using the routes highlighted earlier, were sputter-coated for about 20 min to ensure the deposition of a conducting layer employing a gold target in a JEOL-make unit. The samples were then mounted on a stub that could be inserted through the specimen holder assembly into the chamber of the microscope for viewing at an operating voltage of $20 \mathrm{kV}$. A JEOLmake (JSM 840A) SEM microscope helped first in the characterization of the surface topography and then in the morphological features of the prepared samples. In characterizing these, the experiences gained while studying the homopolymers $^{30}$ were helpful.

Previous effort ${ }^{30}$ concentrated more on homopolymer and block copolymer microscopy than on the present stresses on the comparative situation prevailing in blend and block copolymers, an aspect not reported in the literature using the visual methods. The changes in the surface details along the specimen cross section, if any, were carefully examined, and repetitive features of relevance were photographed. The procedure was also repeated for the case of blended systems. For the ensuing proper interpretation of the scanning micrographs, an exercise of identifying the morphological and surface texture features when only one (homo) phase system is present was undertaken by preparing samples that have either PMMA or PS only as an end product. The physical features of both the block copolymer and the blends were then interpreted with this as a standard reference.

\section{RESULTS AND DISCUSSION}

\section{Characterization and Molecular Weight of PMMAP}

As far as the ${ }^{1} \mathrm{H}$ NMR spectrum for PMMAP is concerned, it showed resonance signals at 1.44 , 


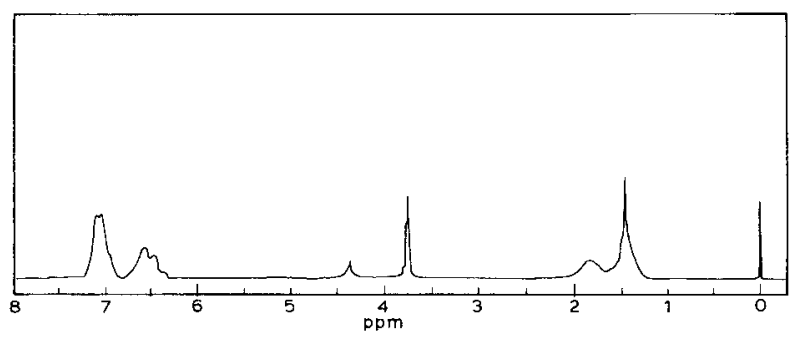

Figure 1. ${ }^{1} \mathrm{H}$ NMR spectrum of PMMAP-PS-PMMAP.

3.76 , and $4.34 \mathrm{ppm}$, which were assigned to $\alpha-\mathrm{CH}_{3},-\mathrm{OCH}_{3}$, and $-\mathrm{OCH}_{2}$ protons, respectively. ${ }^{26}$ The $\bar{M}_{n}$ and $\bar{M}_{w} / \bar{M}_{n}$ values of PMMAP were 3150 and 1.7 , respectively. It should also be noted that the $\bar{M}_{n}$ value is high, and the $\bar{M}_{w} / \bar{M}_{n}$ values are low using the present method compared to 2,2'-azobis(isobutyronitrile) (AIBN)initiated polymerization. ${ }^{15}$

\section{Characterization and Molecular Weight of PMMAP-PS-PMMAP "Active" Polymer}

The ${ }^{1} \mathrm{H}$ NMR spectrum of PMMAP-PS-PMMAP active polymer is displayed in Figure 1. The peaks at $1.44,3.76$, and $4.34 \mathrm{ppm}$ correspond to the $\alpha-\mathrm{CH}_{3},-\mathrm{OCH}_{3}$, and $-\mathrm{OCH}_{2}$ protons, respectively, of PMMAP segments; ${ }^{33}$ on the other hand, those at 1.42, 1.84, and 6.4-7.5 ppm refer to methylene, methine, and aromatic protons of the PS block, ${ }^{34}$ respectively. It may be noted that $\alpha$-methyl and methylene signals at 1.44 and 1.42 ppm of the PMMAP segments and PS block merge into a single peak around $1.4-1.5 \mathrm{ppm}$.

The results derived for bulk polymerization of STY using the macroinitiator PMMAP are presented in Table I. From the tabulated data, it can be seen that the yield (\%) and $\bar{M}_{w} / \bar{M}_{n}$ of the active polymer increase with an increase in concentration of PMMAP. The broader polydispersity can be attributed to the chain-transfer reaction asso-

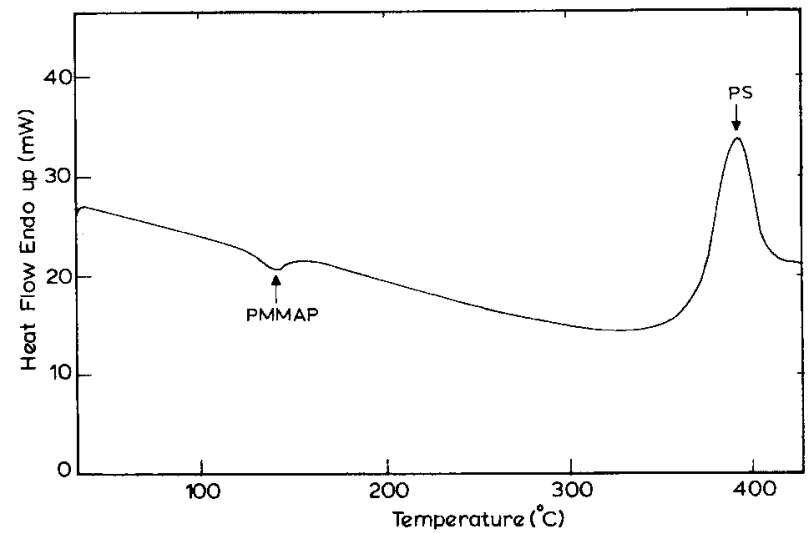

Figure 2. DSC thermogram of PMMAP-PS-PMMAP.

ciated with the degradation products of PMMAP, which in the present experimental condition are formaldehyde and methyl pyruvate, ${ }^{33,35}$ respectively. Of these products, formaldehyde is the chain-transfer agent. ${ }^{30}$

\section{Thermal Analysis of PMMAP-PS-PMMAP "Active" Polymer}

The DSC and TGA traces of PMMAP-PS-PMMAP active polymer are depicted in Figures 2 and 3. The exotherm observed around $140{ }^{\circ} \mathrm{C}$ as well as the broad endotherm observed around $400{ }^{\circ} \mathrm{C}$ (Fig. 2) are ascribed to the decomposition of the PMMAP segments ${ }^{35}$ and PS block, ${ }^{36}$ respectively. The TGA traces (Fig. 3) of this active polymer yield two-step degradation, the first step of which has a small weight loss around $140{ }^{\circ} \mathrm{C}$, followed by a second step where a major loss is registered around $400{ }^{\circ} \mathrm{C}$. These weight losses are attributed to the PMMAP segments and PS block, respectively.

In reference to the position of the peroxy segments in the PS backbone, the PMMAP-PS-PMMAP active polymers were decomposed at $120^{\circ} \mathrm{C}$ for $15 \mathrm{~h}$ in chlorobenzene, following which the $M_{n}$

Table I. Bulk Polymerization of STY with PMMAP at $70{ }^{\circ} \mathrm{C}$ for $3 \mathrm{~h}$

\begin{tabular}{ccccccc}
\hline $\begin{array}{c}{[\text { PMMAP] }} \\
\left(\text { mol-equiv } \cdot \mathrm{L}^{-1}\right)^{\mathrm{a}}\end{array}$ & $\begin{array}{c}{[\mathrm{STY}]} \\
\left(\mathrm{mol} \cdot \mathrm{L}^{-1}\right)\end{array}$ & Yield $(\%)$ & $\bar{M}_{w} \times 10^{-4}$ & $\bar{M}_{n} \times 10^{-4}$ & $\left(\bar{M}_{w} / \bar{M}_{n}\right)$ & $\bar{M}_{n} \times 10^{-4 \mathrm{~b}}$ \\
\hline 0.5 & 8.7 & 8.7 & 9.4 & 2.6 & $(3.6)$ & 2.2 \\
0.8 & 8.7 & 11.2 & 10.3 & 2.4 & $(4.3)$ & 2.0 \\
1.3 & 8.7 & 16.5 & 9.6 & 2.0 & $(4.8)$ & 1.7 \\
\hline
\end{tabular}

a Approximately $1 \mathrm{~mol} \cdot$ equiv is the equivalent weight in grams of the repeat unit

${ }^{b}$ After decomposition of peroxy segments of the active polymers. 


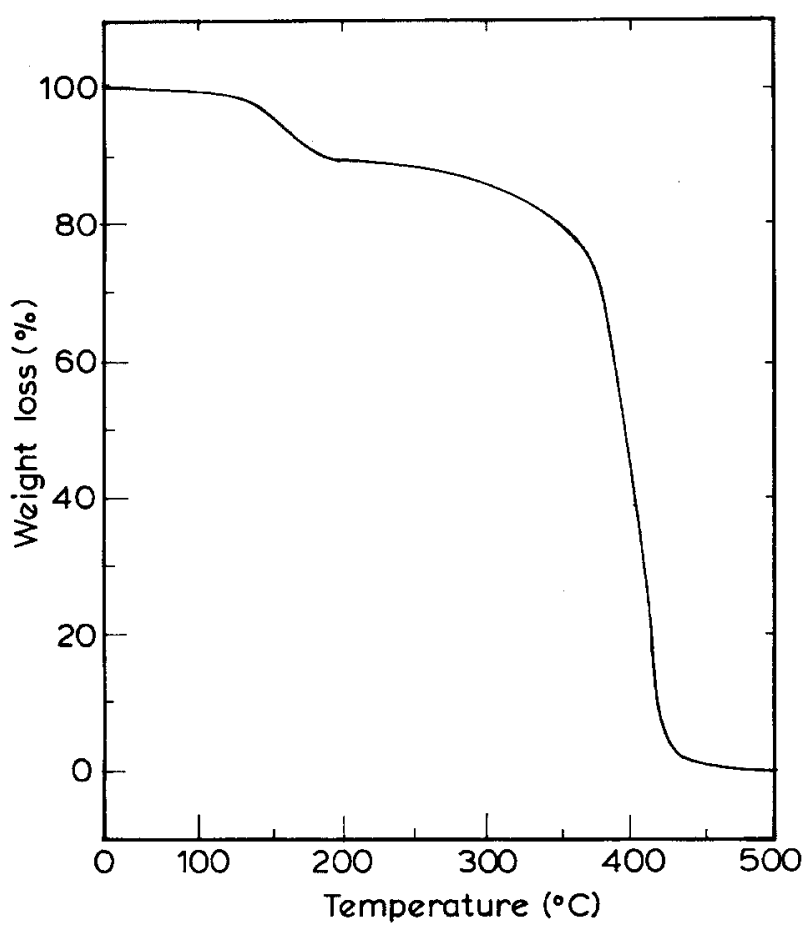

Figure 3. TGA thermogram of PMMAP-PS-PMMAP.

values were calculated. These values are listed in Table I; from the data, it appears that the peroxy segments (i.e., PMMAP segments) are posited in the chain ends of PS, or otherwise a significant reduction in the molecular weight would have been observed.

\section{Mechanism of PMMAP-PS-PMMAP “Active" Polymer}

The occurrence of the PMMAP segments in PS (Fig. 1) can be viewed as a suggestion that PMMAP macroradical, that is, b-peroxy alkoxy radical, formed by the random homolysis of the peroxide linkage, is responsible for the initiation of STY polymerization. The propagating radicals of PS, however, undergo bimolecular termination by radical recombination with the PMMAP seg- ments to yield the PMMAP-PS-PMMAP active polymer.

\section{Metamorphosis of Block-into-Block Copolymers}

While it is recognized that block copolymers may be synthesized using free radicals, ${ }^{37}$ that for the metamorphosis of a block copolymer macroinitiator like PMMAP-PS-PMMAP into another block copolymer like PMMA-b-PS-b-PMMA is an area that is quite interesting and less explored. ${ }^{27,28} \mathrm{By}$ using PMMAP-PS-PMMAP as a thermal initiator, PS was converted into another block copolymer like PMMA-b-PS-b-PMMA by the degradation of peroxide segments in the presence of monomer MMA. The results for the block copolymerization of MMA using active polymer PMMAP-PS-PMMAP are recorded in Table II. From this tabulation it appears that PMMA-b-PS-bPMMA show narrow polydispersity compared to the active polymer PMMAP-PS-PMMAP. This observation points out the concentration of degradation products is very small, owing to the presence of less proportion of peroxy segments in the chain ends. Consequently, chain-transfer reactions are drastically reduced, owing to the decrease in polydispersity.

\section{${ }^{1}$ H NMR Spectrum of PMMA-b-PS-b-PMMA}

Reconfirming the spectra results reported earli$\mathrm{er},{ }^{30}$ the ${ }^{1} \mathrm{H}$ NMR spectrum of the present system showed the characteristic signals of PS as 1.4 ( $-\mathrm{CH}_{2}$-), 1.84 (>CH-), 6.4-6.6 (o-protons), and $7.2 \mathrm{ppm}$ ( $p$ - and $m$-protons); and of PMMA as 0.8-1.1 $\left.\left(-\mathrm{CH}_{3}\right), 1.89\left(-\mathrm{CH}_{2}\right)^{-}\right)$and $3.65 \mathrm{ppm}$ $\left(-\mathrm{OCH}_{3}\right)$, thereby confirming the formation of PMMA-b-PS-b-PMMA. The absence of characteristic PMMAP signals in the ${ }^{1} \mathrm{H}$ NMR spectrum of PMMA-b-PS-b-PMMA shows the lack of any residual PMMAP segments in the backbone (Fig. 4). Furthermore, from the peak integration ratio of $-\mathrm{CH}_{2}$ protons of PS and PMMA blocks, it can be

Table II. Block Copolymerization with MMA by PMMAP-PS-PMMAP at $75^{\circ} \mathrm{C}$ for $5 \mathrm{~h}$

\begin{tabular}{ccccrr}
\hline $\begin{array}{c}\text { PMMAP-PS-PMMAP } \\
(\text { wt \%) }\end{array}$ & $\begin{array}{c}{[\mathrm{MMA}]} \\
\left(\mathrm{mol} \cdot \mathrm{L}^{-1}\right)\end{array}$ & Yield (\%) & $\begin{array}{c}\text { Block Copolymer } \\
(\text { wt \%) }\end{array}$ & $\bar{M}_{n} \times 10^{-4}$ & $\bar{M}_{w} / \bar{M}_{n}$ \\
\hline 8.5 & 9.4 & 20.5 & 43.3 & 10.5 & 2.2 \\
13.7 & 9.4 & 28.9 & 44.6 & 9.7 & 2.5 \\
\hline
\end{tabular}




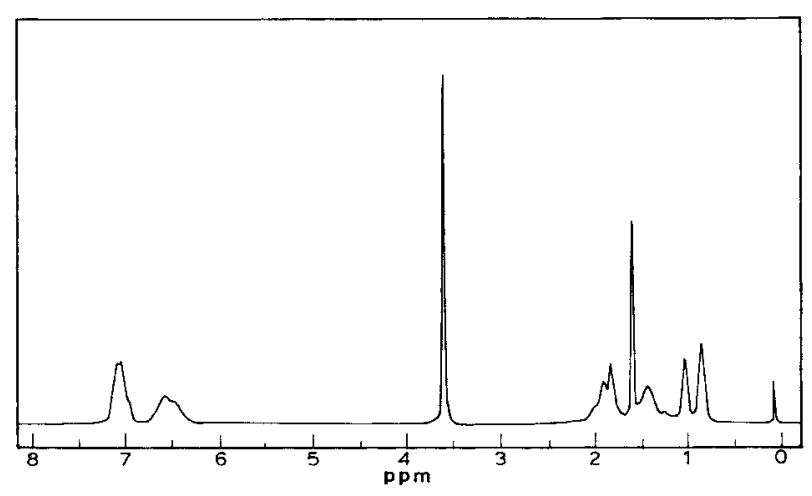

Figure 4. ${ }^{1} \mathrm{H}$ NMR spectrum of PMMA-b-PS-bPMMA.

inferred that the block copolymer contains $25: 75$ proportions of PS and PMMA blocks, respectively.

\section{Thermal Analysis of PMMA-b-PS-b-PMMA}

TGA of PMMA-b-PS-b-PMMA revealed two-step weight loss. The first step of degradation occurred around $350{ }^{\circ} \mathrm{C}$, which referred to the degradation of the PMMA block, and the second step of degradation that took place at $400{ }^{\circ} \mathrm{C}$ corresponded to the PS block similar to the one encountered earlier. ${ }^{30}$ Therefore, the absence of any weight loss at $140{ }^{\circ} \mathrm{C}$ reconfirms the absence of any residual PMMAP segments in the block copolymer (referred to in the NMR spectra analysis earlier).

\section{Mechanism of PMMA-b-PS-b-PMMA Formation}

The mechanism for the formation of PMMA-b-PSb-PMMA using PMMAP-PS-PMMAP active polymer is presented in Scheme 1. The PMMAP-PSPMMAP on heating generated b-peroxy alkoxy radicals A and B (eq 1 in Scheme 1). The radical "B" further undergoes dissociation producing $\operatorname{poly}(\alpha$ styrenyl dioxy) ( $\cdot \mathrm{OPSO} \cdot)$ and b-peroxy alkoxy radical $\mathrm{A}^{\prime}$ (eq 3). The alkoxy radical $\mathrm{A}\left(\mathrm{A}^{\prime}\right)$ may undergo a chain-unzipping reaction forming methyl pyruvate and formaldehyde (eq 4) or react with MMA emanating in the generation of the PMMA homopolymer (eq 5). The - OPSO - thus formed adds to MMA, resulting in the formation of the PMMAb-PS-b-PMMA block copolymer (eq 6).

\section{Thermochemical Calculations}

To further understand the mechanism of polymerization, thermochemical studies on the heats of formations $\left(\Delta H^{\circ}\right)$ of monomers and various rad- icals have been executed. Here, only the model radicals instead of actual macroradicals have been considered. The commercial semiempirical program packages, MOPAC version 6.0, were used for all computations. ${ }^{38}$ The geometry of all the radicals were fully optimized using the AM1 Hamiltonian.

The calculations show that the unzipping of alkoxy radicals to nonradical products (eq 4) is thermodynamically more favorable [heat of reaction $\left(\Delta H_{r}\right)=-315.8 \mathrm{~kJ} \mathrm{~mol}^{-1}$ ] than addition to the monomer $\left(\Delta H_{r}=-190.7 \mathrm{~kJ} \mathrm{~mol}^{-1}\right)$ (eq 5).

Evans-Polanyi ${ }^{39}$ have proposed an empirical relationship where the exothermicity and activation energy in radicals/atoms in a series of closely related compounds are covered. If the effects due to stabilization of the transition state are the same, an increase in the heat of exothermic reaction by an amount $\Delta H_{r}$ will cause a decrease in the activation energy $(\Delta E) .{ }^{39,40}$ Hence, kinetically too, the latter process requires more activation energy than the former, that is, unzipping reaction, ${ }^{41}$ therefore, the unzipping process (eq 4) is much faster than the addition of the alkoxy radicals to the double bond (eq 5). ${ }^{41}$

\section{Characterization of Blend}

The blend after purification was characterized by ${ }^{1} \mathrm{H}$ NMR spectroscopy. From such an approach, the peak integration ratio of PMMA and PS was found to be $75: 25$. In other words, it was ensured that the proportions of the two polymeric constituents in the system remained nearly identical. Microscopic characterization of the products obtained through the two different routes is taken out in the following section.

\section{Morphological Characterization}

Emphasis here is given to the morphological details as well as the surface texture of the constit-

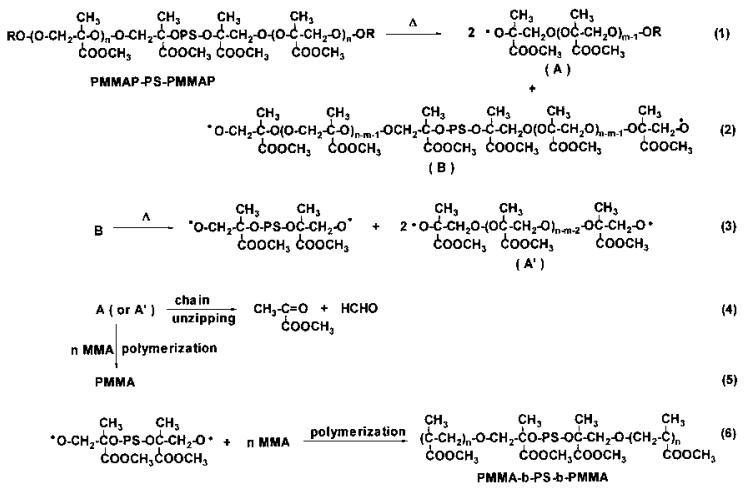

Scheme 1 
uent phase systems (i.e., PMMA and PS). As stated in the experimental section, the interpretation of the features was facilitated by compiling extensive SEM databases when only one phase system (PMMA or PS) is present and then comparing the scanning micrograph obtained on the products of the differently processed routes with the developed database. This exercise with monophase systems showed that for the case when only PS is present, the photographs yielded an irregular contour, undulating terrain-bearing surface that had porous sites on them as well as evidence of sprinkling of particles. ${ }^{30}$ The PMMA system, on the other hand, yielded a smooth nearconstant cross-sectional area bearing threadlike features that show an occasional twist or curl or U-bend. ${ }^{30}$

Considering the case of the blended category first, a lower magnification view, where PMMA, having a smooth surface and sandwiched between PS bearing materials, marked by letter $\mathrm{S}$ can be seen in Figure 5(a). Also visible are the two interface regions forming the boundary between the PS and PMMA (the latter marked by letter M) regions. A feature noticed in this blend is that PS has a rough surface with a contour showing many protrusions [as, for instance, at $\mathrm{P}$ in Fig. 5(b)]. Further, in the same photograph [Fig. 5(b)] the rough terrain exhibited by the surface of the PS bearing region (for instance, at regions marked by letter T) is very distinct.

As a result, it may be said that the blend has a smoother PMMA, containing regions of PS adjacent to it and where the PS has either the appearance of a surface distributed with dispersed particles or what can be categorized as porous, rough texture. Also, the fact that a distinct interphase interface region can be seen between the PS- and PMMA-juxtaposed regions emerges from the present article. Having examined the blends, the features seen in the block copolymer case are now considered.

Thus, the features, wherein the block copolymer displays a bend at a region marked $\mathrm{K}$ [following which the surface of the material shows rough protruded and cross-sectional-wise expanded features (at $\mathrm{S}$ )] typically seen in the PS regions, are shown in Figure 6(a).(FIG6\} Figure 6(a) also shows smooth PMMA (marked P on the left side) leading to a region where rough-surface-bearing PS (marked S) exists. This feature of PMMA leading to a differently appearing morphological material is best seen in Figure 6(b), ${ }^{30}$ where at the region marked $\mathrm{M}^{\prime}$ a trident-shaped PMMA
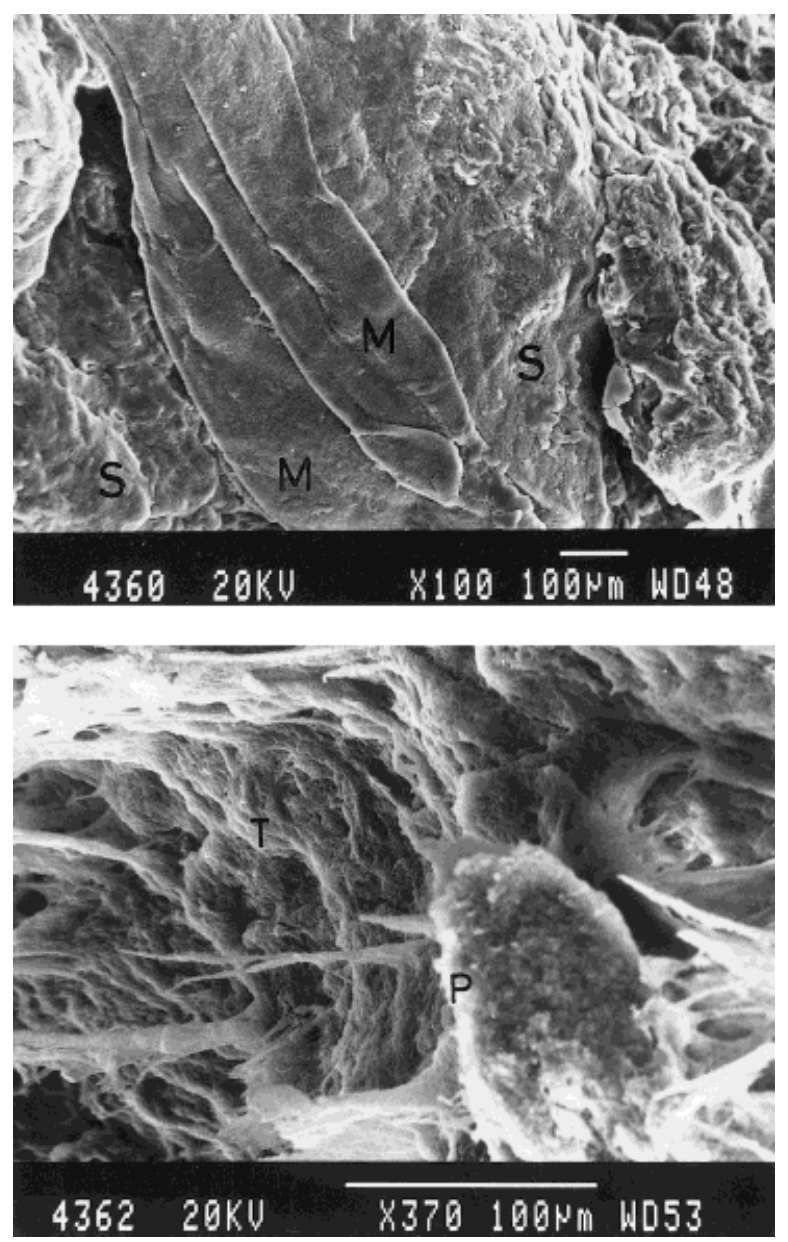

Figure 5. (a). The rough surface feature of PS-bearing regions posited on either side of asmooth surface bearing PMMA. (b). The surface feature of a PS showing very uneven terrain as well as the edge displaying protrusions.

emerges. One branch of this trident (the top marked $\mathrm{M}^{\prime}$ ) leads to a broadened and expanded region whose surface appears to be irregular (marked $\mathrm{S}^{\prime}$ ). The central thick branch marked $\mathrm{M}^{\prime \prime}$ also leads to a wider region marked $\mathrm{S}^{\prime \prime}$. The third branch at the lower wing marked $\mathrm{M}^{\prime \prime \prime}$ leads to a region where at the point marked $\mathrm{K}$ a wellformed, irregular, porous and rough-terrain-bearing PS region marked $\mathrm{S}^{\prime \prime \prime}$ may be seen.

As a result, it is of interest to note that the PS component of the micrograph displayed a typically rough irregular surface similar to what was seen in the case of the blended material. The distinction between the two differently processed but bearing the same volume fractions of polymer materials surfaces when the PS part of the block copolymer case has been considered. Here, the PS 

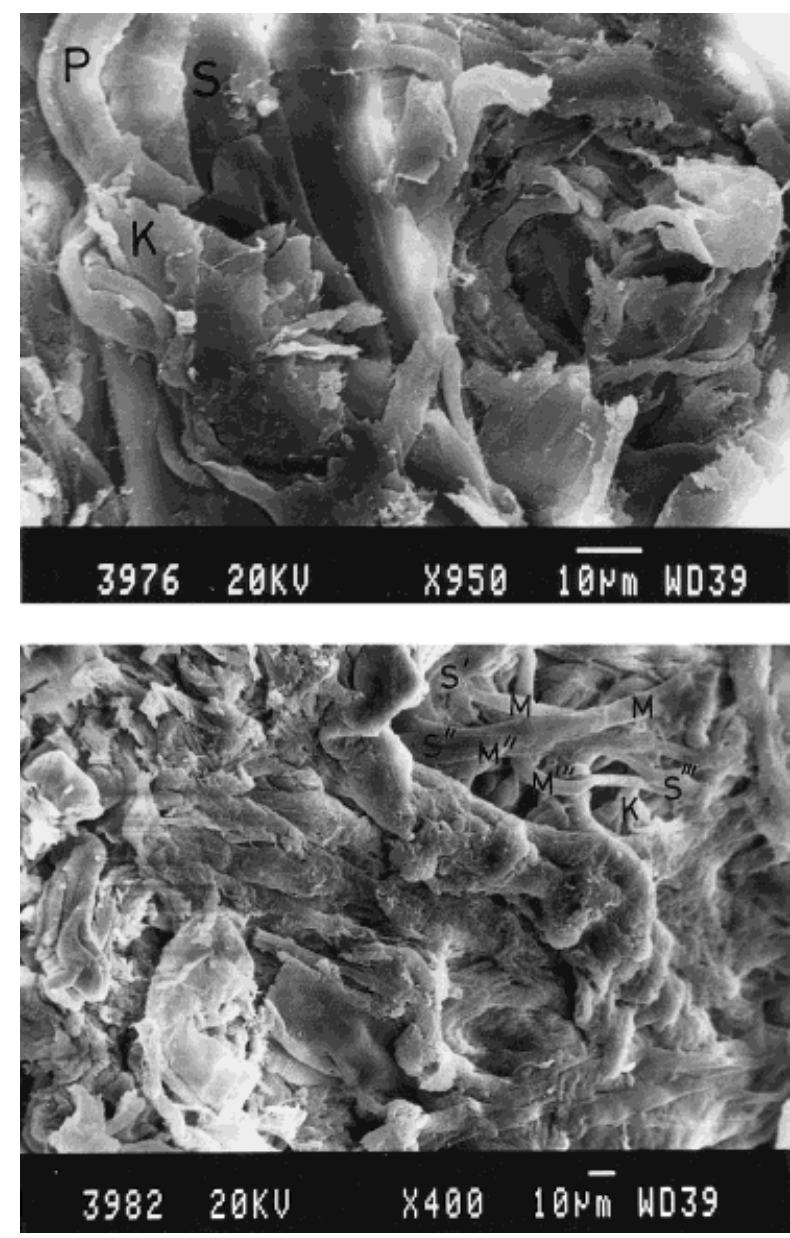

Figure 6. (a). SEM micrograph showing both PMMA and PS bearing regions in block copolymer. (b). Scanning micrograph depicting the branching segments segments of PMMA and leading to PS containing regions. The photograph also displays the distribution of PS along with PMMA.

segment is continuous with PMMA. Furthermore, the porous nature of the surface on PS, seen earlier with the blended portion, is conspicuous by its poor presence in the scanning micrograph for the block copolymer case. In addition to these, whereas in blends the interface between the PS and PMMA regions are distinct, a similar feature is difficult to see in the block copolymer. Thus, a distinct difference both in the surface texture and distribution of the porous sites in PS occurs for the blends together with a block copolymer. Also, whereas a distinguishable interface between the two polymeric materials exists for the former, the same is not the case, expectedly, for the latter. The results re-emphasize the well-known fact that the processing route contributes in a signif- icant way to the appearance of the structure as seen in SEM.

\section{CONCLUSION}

The thermal-initiating potentialities of PMMAP for the synthesis of active polymer PMMAP-PSPMMAP have been demonstrated. The efficiency of the active polymer has been measured by the synthesis of block copolymer PMMA-b-PS-bPMMA. Also, noticeable differences in the surface texture and morphology of the block copolymer together with the blend case exist. Thus, the blended material showed a distinguishable interfacial region between two homopolymers, and the PS material that displayed a rough surface with a contour showing many protrusions for the block copolymer case yielded two homopolymers that are continuous and without a well-demarcated interface region. Further, the surface roughness exhibited for the PS part of the block copolymer is much less prominently seen.

In summary, vinyl polyperoxides are potentially inexpensive materials because they can be made by reacting a vinyl monomer with air. Also, their synthesis is simple and handling is less hazardous compared to acid polyperoxides. All of these considerations suggest that vinyl polyperoxides can be used as initiators.

\section{REFERENCES AND NOTES}

1. European Chemical News, 20 February 1989.

2. Hedrick, J. L.; Carter, K. R.; Labadie, J. W.; Miller, R. D.; Volksen, W.; Hawker, C.; Yoon, D. Y.; Russel, T. P.; McGrath, J. E.; Briber, R. M. Adv Polym Sci 1999, 141, 1.

3. Bates, F. S. Science 1991, 251, 898.

4. Yamashita, Y. In Chemistry and Industry of Macromonomers; IPC: Tokyo, 1984; p 1.

5. Kohler, J.; Reiss, G.; Banderet, A. Eur Polym J 1986, 4, 187.

6. Nyhus, A. K.; Hagen, S.; Berge, A. J Polym Sci Part A: Polym Chem 1999, 37, 3973.

7. Matheswaran, M.; Padmanabhan, K.; Kishore J Mater Sci Lett 1995, 14, 951.

8. Chandra, D. S.; Rao, R. M. V. G. K.; Kishore J Mater Sci Lett 1991, 10, 1263.

9. Kishore; Venkatraman, S. J Appl Polym Sci 1998, 67,65 .

10. Folkes, M. J.; Reip, P. W. Polymer 1986, 27, 377.

11. Aggarwal, S. L. In Processing, Structure and Properties of Block Copolymers; Folkes, M. J., Ed.; Ap- 
plied Science Publishers Ltd.: London, 1985; Chapter 1.

12. Han, C. D. Multiphase Flow in Polymer Processing; Academic Press: New York, 1981.

13. Lyngaae-Jorgensen, J. In Processing, Structure and Properties of Block Copolymers; Folkes, M. J., Ed.; Applied Science Publishers Ltd.: London, 1985; Chapter 3.

14. Simonescu, C. L.; Comanita, E.; Pastravanu, M.; Dimitriu, S. Prog Polym Sci 1986, 12, 1.

15. Mukundan, T.; Kishore, K. Prog Polym Sci 1990, 15,475 .

16. Kishore, K.; Ravindran, K. Macromolecules 1991, $15,1638$.

17. Nanda, A. K.; Kishore, K. Polymer, in press.

18. Nanda, A. K.; Kishore, K. Indian J Chem 2000, $39 \mathrm{~A}, 631$.

19. Kishore, K.; Mukundan, T. Nature (London) 1986, $324,130$.

20. Mukundan, T.; Annakutty, K. S.; Kishore, K. Fuel 1992, 72, 688 .

21. Nanda, A. K.; Kishore, K. J Appl Polym Sci, in press.

22. Subramanian, K.; Kishore, K. Eur Polym J 1997, 33, 1365.

23. Mukundan, T.; Kishore, K.; Bhanu, V. A. J Chem Soc Chem Commun 1989, 12, 781.

24. Hazar, B.; Baysal, B. M. Polymer 1986, 27, 961.

25. Yilgor, I.; Baysal, B. M. Makromol Chem 1985, 186, 463.

26. Ivanchev, S. S. Russ Chem Rev 1991, 60(7), 689.
27. Yamamoto, T.; Aoshima, K.; Ohmura, H.; Moriya, Y.; Suzuki, N.; Oshibe, Y. Polymer 1991, 32, 19.

28. Endo, K.; Murata, K.; Otsu, T. Polymer 1992, 33, 3976.

29. Perrin, D. D.; Armarego, N. L. F. Purification of Laboratory Chemicals; Pergamon Press: Oxford, 1988.

30. Nanda, A. K.; Kishore J Polym Sci Part A: Polym Chem 2000, 38, 3665.

31. Sankarpandian, M.; Kishore, K. Macromolecules 1994, 27, 7278.

32. Sankarpandian, M.; Kishore, K. Macromolecules 1991, 24, 3090.

33. Mukundan, T.; Kishore, K. Macromolecules 1987, 20, 2382.

34. Pham, Q.; Petiaud, R.; Waton, H. Proton and Carbon NMR Spectra of Polymers; Wiley: New York, 1983.

35. Nanda, A. K.; Ganesh, K.; Kishore, K.; Surinarayan, M. Polymer 2000, 41, 9063.

36. Jang, L. W.; Lee, D. C. Polymer 2000, 41, 1749.

37. Quirk, R. P.; Kinning, D.; Fetters, L. J. In Comprehensive Polymer Science; Pergamon Press: New York, 1989; Vol. VII, Chapter 1, pp 1-26.

38. Dewar, M. J. S.; Zeobisch, E. G.; Healy, E. F.; Stewart, J. J. P. J Am Chem Soc 1985, 107, 3902.

39. Kochi, J. K. Free Radicals; Wiley-Interscience: New York, 1973; Vol I; pp 26, 283, and 288.

40. Sawada, H. Thermodynamics of Polymerization; Marcel Dekker: New York, 1976; p 101.

41. Mayo, F. R. J Am Chem Soc 1958, 2465, 1958. 